\title{
A BOUNDARY CONDITION FOR THE VANISHING OF $n$ HOLOMORPHIC FUNCTIONS IN COMPLEX $n$-SPACE
}

\section{J. J. KOHN ${ }^{1}$}

In this note we prove that if $f_{1}, \cdots, f_{n}$ are holomorphic functions on $B$, the unit ball in complex $n$-space, and if

$$
\sum_{\alpha=1}^{n} \bar{z}_{\alpha} f_{\alpha}=0
$$

on the boundary of $B$, then $f_{1}=0, f_{2}=0, \cdots, f_{n}=0$ throughout $B$. We assume that the $f_{\alpha}$ are continuous in the closure of $B$.

The above theorem can be considered as a special case of a boundary value problem for forms of type $(1,0)$ on a finite Kähler manifold (see [1]). Namely, let

$$
\psi=\sum_{\alpha=1}^{n} f_{\alpha} d z_{\alpha}
$$

and

$$
\Phi=\sum_{\beta=1}^{n} z_{\beta} d \bar{z}_{\beta} ;
$$

then if condition (A) is satisfied and if

$$
\Delta(\psi \wedge \Phi)=0
$$

(where $\Delta$ is the laplacian

$$
\Delta=-4 \sum_{v=1}^{n} \frac{\partial^{2}}{\partial z_{v} \partial \bar{z}_{v}}
$$

which on differential forms acts separately on each component) it follows that $\psi$ is zero. Note that condition (A) is a type of contraction of $\psi$ with $\bar{\Phi}$ and that (a) is necessary and sufficient for the holomorphy of the $f_{\alpha}$. Further note that $d \Phi$, the exterior derivative of $\Phi$, is the form associated with the Kähler metric on $B$.

Proof of Theorem. Let

Received by the editors August 22, 1957.

${ }^{1}$ This work was supported by Office of Ordnance Research Contract No. DA 36-034-ORD-2164. 


$$
B=\left\{\left.\left(z_{1}, \cdots, z_{n}\right)\left|\sum_{\alpha=1}^{n}\right| z_{\alpha}\right|^{2}<1\right\}
$$

and

$$
S=\left\{\left.\left(z_{1}, \cdots, z_{n}\right)\left|\sum_{\alpha=1}^{n}\right| z_{\alpha}\right|^{2}=1\right\} .
$$

The subspace in complex $n$-space orthogonal to the vector $\left(\bar{z}_{1}, \bar{z}_{2}, \cdots, \bar{z}_{n}\right)$ is spanned by the vectors

$$
\begin{array}{lc}
A_{1}=\left(\bar{z}_{2},-\bar{z}_{1}, 0, \cdots, 0\right), \\
A_{2}=\left(\bar{z}_{3}, 0,-z_{1}, \cdots, 0\right), \\
\vdots & \vdots \\
A_{n-1}=\left(\bar{z}_{n}, 0, \cdots, 0,-\bar{z}_{1}\right),
\end{array}
$$

so that condition (A) implies that there exist functions $\lambda_{1}, \cdots, \lambda_{n-1}$ on $S$ such that

$$
\left(f_{1}, \cdots, f_{n}\right)=\lambda_{1} A_{1}+\lambda_{2} A_{2}+\cdots+\lambda_{n-1} A_{n-1} .
$$

Writing this by components we get

$$
\begin{aligned}
& f_{1}=\lambda_{1} \bar{z}_{2}+\lambda_{2} \bar{z}_{3}+\cdots+\lambda_{n-1} \bar{z}_{n}, \\
& f_{2}=-\lambda_{1} \bar{z}_{1}, \\
& f_{3}=-\lambda_{2} \bar{z}_{1}, \\
& \dot{f_{n}}=-\lambda_{n-1} \bar{z}_{1} .
\end{aligned}
$$

So on $S$ we have

$$
f_{\alpha}=-\lambda_{\alpha-1} \bar{z}_{1} \quad \text { for } \alpha=2,3, \cdots, n .
$$

Multiplying by $z_{1}$, we get

$$
z_{1} f_{\alpha}=-\lambda_{\alpha-1}\left|z_{1}\right|^{2}=-\lambda_{\alpha-1}\left(1-\left|z_{2}\right|^{2}-\cdots-\left|z_{n}\right|^{2}\right) .
$$

Hence if $z_{1} \neq 0$

$$
\lambda_{\alpha-1}=\frac{-z_{1} f_{\alpha}}{1-\left|z_{2}\right|^{2}-\cdots-\left|z_{n}\right|^{2}} .
$$

Thus the $\lambda_{\beta}$ can be extended to functions on $B-\left\{\left(z_{1}, \cdots, z_{n}\right) \mid z_{1}\right.$ $=0\}$ which are holomorphic in $z_{1}$. Now differentiating $\lambda_{\alpha-1}$ with respect to $\bar{z}_{\beta}$ we obtain 


$$
\frac{\partial \lambda_{\alpha-1}}{\partial \bar{z}_{\beta}}=\left\{\begin{array}{cc}
0 & \text { if } \beta=1 \\
\frac{-z_{1} z_{\beta} f_{\alpha}}{\left(1-\left|z_{2}\right|^{2}-\cdots-\left|z_{n}\right|^{2}\right)^{2}} & \text { if } \beta>1
\end{array}\right.
$$

on $B-\left\{z \mid z_{1}=0\right\}$.

Differentiating $f_{1}$ with respect to $\bar{z}_{\beta}$ :

$$
0=\frac{\partial \lambda_{1}}{\partial \bar{z}_{\beta}} \bar{z}_{2}+\frac{\partial \lambda_{2}}{\partial \bar{z}_{\beta}} \bar{z}_{3}+\cdots+\frac{\partial \lambda_{n-1}}{\partial \bar{z}_{\beta}} \bar{z}_{n}+\lambda_{\beta-1} \quad \text { for } \beta>1 .
$$

Substituting the expression for $\partial \lambda_{\alpha-1} / \partial \bar{z}_{\beta}$ into the above equation we obtain

$$
0=\frac{-z_{1} z_{\beta}}{\left(1-\left|z_{1}\right|^{2}-\cdots-\left|z_{n}\right|^{2}\right)^{2}}\left[\bar{z}_{2} f_{2}+\cdots+\bar{z}_{n} f_{n}\right]+\lambda_{\beta-1} .
$$

Evaluating on $S$ by use of condition (A)

$$
z_{\beta} f_{1}+\left|z_{1}\right|^{2} \lambda_{\beta-1}=0 .
$$

But since

$$
\bar{z}_{1} \lambda_{\beta-1}=-f_{\beta}
$$

we obtain

$$
z_{\beta} f_{1}=z_{1} f_{\beta} .
$$

Multiplying by $\bar{z}_{\beta}$ and summing over $\beta$

$\left(\left|z_{2}\right|^{2}+\left|z_{3}\right|^{2}+\cdots+\left|z_{n}\right|^{2}\right) f_{1}=z_{1}\left(\bar{z}_{2} f_{2}+\cdots+\bar{z}_{n} f_{n}\right)=-\left|z_{1}\right|^{2} f_{1}$.

Adding $\left|z_{1}\right|{ }^{2} f_{1}$ to both sides and evaluating on $S$ we get

$$
f_{1}=0 \text {. }
$$

Similarly by appropriate choices of bases for vectors orthogonal to $\left(\bar{z}_{1}, \cdots, \bar{z}_{n}\right)$ we get $f_{2}=0, f_{3}=0, \cdots, f_{n}=0$. Q.E.D.

\section{REFERENCE}

1. J. J. Kohn and D. C. Spencer, Complex Neumann problems, Ann. of Math. vol. 65 , no. 4 (1957).

Princeton University 